\title{
Opportunity trajectory reconstruction techniques for evaluation of ATC systems
}

\author{
JESUS GARCIA, JUAN A. BESADA, ANDRES SOTO AND GONZALO DE MIGUEL
}

\begin{abstract}
This paper describes some key points of a new tool being currently developed by Eurocontrol for the assessment of air traffic control (ATC) multisensor trackers performance. It sumwarizes the algorithmic foundations of the high-accuracy trajectory reconstruction process used to obtain reference trajectories from recorded weasures. These trajectories will serve as a reference for the evaluation of the accuracy of ATC data processing centers. The performance of the system is illustrated with some reconstruction experiments on synthetic and real data.
\end{abstract}

Keywords: Air traffic control, data fusion, sensor fusion, smoothing, tracking assessment, trajectory reconstruction

\section{INTRODUCTION}

Trajectory Reconstruction and Evaluation Suite (TRES) will become in the near future a replacement for some parts of current versions of Surveillance Analysis Support System for Centers (SASS-C) suite [1]. This is a system used for the performance assessment of air traffic control (ATC) multisensor/ multitarget trackers. This paper describes the overall architecture of the assessment system, and details some of its most innovative elements related to opportunity trajectory reconstruction (OTR).

OTR is a batch process within TRES where all the available real data from all available sensors are used in order to obtain smoothed trajectories for all aircraft in the interest area. It requires accurate measure-to-reconstructed trajectory association, bias estimation and correction to align different sensor measures, and adaptive multisensor smoothing to obtain the final interpolated trajectory. It should be pointed out that it is an off-line batch processing potentially quite different from usual real-time data fusion systems used for ATC. Data processing order and specific processing techniques will be different.

In fact, one of the main uses of TRES is the evaluation of the performance of real-time multisensor-multitarget trackers used for ATC, when they are provided with the same measurements as TRES. OTR works as a special multisensor fusion system, aiming to estimate target kinematic state, in which we take advantage of the knowledge of future target position reports (smoothing). TRES must be able to process the following kinds of data:

- Radar data, from primary (PSR), secondary (SSR), and mode $S$ radars, including enhanced surveillance. These measurements have random errors in the order of hundreds of meters (with size increasing linearly with distance to radar), and they may produce losses of detection and false alarms. They also suffer large systematic errors (biases). With enhanced surveillance, mode $S$ radars may provide velocity information.

- Multilateration data from wide area multilateration (WAM) sensors. They have much lower errors (in the order of 5-100 m), also increasing with distance to sensor coverage center, but in a complex manner depending on the receiving stations situation relative to the target.

- Automatic dependent surveillance-broadcast (ADS-B) data. Its quality is dependent on aircraft equipment, but with the current trend to adopt GPS/GNSS, it will have errors in the order of 5-20 m. In ADS-B measures there could be systematic errors due to incorrect time stamping. This sensor may also provide velocity information.

The complementarity nature of these sensor techniques allows a number of benefits (high degree of accuracy, extended coverage, systematic errors estimation, correction, etc.) and brings new challenges for the fusion process in order to guarantee an improvement with respect to any of the sensor techniques used alone. An important novelty is the integration of traditional ground-based surveillance (PSR/SSR radars) with modern sensors such as WAM, with increased accuracy, and airborne sensors providing extended detection capability (velocity and maneuvers). The fusion of all measurements requires new solutions and a robust process that considers detailed characteristics of all data sources and checks their consistency before being fused.

The two basic aspects for estimating the reference trajectory are the development of appropriate models for sensor errors and target behavior. As mentioned, the model of sensor errors should address the probability density function (systematic and random components), to be exploited in the reconstruction process. Regarding the model of target behavior, in this work we propose a model-based reconstruction, based in a practical implementation of the optimal smoother [2] and taking advantage of physical motion models tuned for aircraft flying in controlled airspace. Those aircraft follow quite regular patterns, although at the same time the 
system also has to consider more irregular flights in other areas.

In consequence, the paper is divided into two main parts. After a description of the general trajectory reconstruction architecture (Section II), we devote one section to the problem of systematic error correction and association (Section III) and another to the reconstruction (smoothing) algorithms (Section IV). Finally, we conclude the paper with the presentation of some illustrative results (Section V).

\section{DTR ARCHITECTURE}

OTR main architecture is composed of the processes explained below (which are executed sequentially with the order of the following list): the OTR system follows the steps below to generate reference trajectories:

- Converting all measurements to the same coordinates system and correct user provided systematic errors of each sensor. The measurements of each sensor are converted from its measured coordinates system to stereographic central coordinates. If information about sensor measurement variances exists, it is also converted to the noise covariance matrix in central coordinates.

- Selecting the data for trajectory reconstruction applying filters specified by the TRES user.

- Gross association, adequate for batch processing track reconstruction procedures. The proposed solution is based on the use of a conservative association procedure to be used for sensor noise and bias estimation, and a refinement of such association, after bias correction, for reconstruction (smoothing) and later steps. Gross association is the name given to the first process, similar to a realtime multisensor-multitarget association system.

- Noise variance and bias estimation for radar, WAM, and ADS methods using measurements (real traffic and fixed field transponder, if it exists). There could be inaccuracies in the noise parameters provided by the user, and hence overall system robustness and precision will be enhanced with the estimation of sensor noise covariance.

- Fine association refines the process in gross association after sensor bias correction. It contains methods for recovering data discarded during gross association and methods to enhance reconstruction continuity over coverage gaps.

- Trajectory reconstruction based on a particular implementation of the optimal smoother adapted to the aircraft local mode of flight (MoF) preliminary forward-backward MoF segmentation.

\section{ASSDCIATIDN AND BIAS ESTIMATION}

\section{A) Gross association}

Gross association is performed in two parallel processes:

- Monosensor track association: It is different depending on sensor type, running in parallel for all sensors.

- Multisensor track association: It uses monosensor tracks, resampled at common times, to merge them in a multisensor track.
Specific monosensor association processes will be used for each kind of sensor, performing the following:

- Target code correlation if this information is available (not for PSR).

- Barometric and geometric height compatibility test if those measurements are available (different sensors provide different kinds of data).

- Space/time compatibility test, based on Kalman filters and gating tuned to sensor error characteristics.

The monosensor association procedure is quite conservative, closing tracks in potentially problematic association situations. Fine association will be able to recover from this situation.

Multisensor association is performed by synchronous resampling at a low rate of monosensor tracks (both position and velocity) and correlation of codes and of several samples. Only long (stable) tracks will be created in these first stages of the algorithm, as only long monosensor tracks will be allowed to be used in multisensor track association.

In this stage, we also perform a detection of nonmaneuvering segments, to be exploited for bias and noise estimation systems.

\section{B) Noise and bias estimation}

Much effort has been devoted in the last years to the definition of bias estimation procedures for multisensor-multitarget tracking systems (among others $[3-5]$ ). These efforts have been mainly concentrated, in ATC environments, on radar bias estimation and correction, as they are the most widely used sensors for this application.

With the advent of new families of sensors, such as ADS-B, WAM, or high-resolution airport radars, the means for bias estimation must be extended.

The proposed procedures are based on the definition of a series of error models for noise and bias estimation for the different sensors previously described.

\section{1) ATC RADAR ERROR MODELS}

There are mainly two types of radars used in ATC, primary (PSR) and secondary (SSR and mode S) radars. They measure range and azimuth, and in the case of SSR or mode $S$, they also receive height from the aircraft barometer.

In the mode $S$ and conventional secondary radar error model, $k$ th range-azimuth measurement $\left(R_{k}, \theta_{k}\right)$ includes the terms in (1):

$$
\begin{aligned}
& R_{k}=(1+K) R_{i d}(k)+\Delta R+\Delta R_{j}+n_{R}(k), \\
& \theta_{k}=\theta_{i d}(k)+\Delta \theta+\Delta \theta_{1} \sin \theta_{i d}(k)+\Delta \theta_{2} \cos \theta_{i d}(k)+n_{\theta}(k),
\end{aligned}
$$

where $\left(R_{i d}(k), \theta_{j, d}(k)\right)$ are the ideal target positions for the $k$ th measurement, expressed in local polar coordinates, $\Delta R$ is the radar range bias, $K$ is the gain of range bias, $\Delta R_{j}$ is the transponder-induced bias of the $j$ th aircraft, different for each aircraft, $\Delta \theta$ is the azimuth bias, $\left(\Delta \theta_{1}, \Delta \theta_{2}\right)$ are the values that characterize the radar's azimuth eccentricity, and $\left(n_{R}(k), n_{\theta}(k)\right)$ are the measurement noise errors.

Primary radar has the same model, except for the lack of the $\Delta R_{j}$ term. 
When we translate this measure to the stereographic plane, we use a quite exact non-linear coordinate transformation method like the one used by Paradowski and Kowalski [6]. This method implements a function we call $f_{\text {Radar }}(\cdot)$. So, to project error terms into the stereographic plane, we make a first-order approximation of this transformation, and have

$$
\begin{gathered}
{\left[\begin{array}{l}
x_{k} \\
y_{k}
\end{array}\right]=f_{\text {Rudar }}\left(\left[\begin{array}{l}
R_{k} \\
\theta_{k}
\end{array}\right]\right) \cong\left[\begin{array}{l}
x_{i d}(k) \\
y_{i d}(k)
\end{array}\right]+H_{R}\left[\begin{array}{c}
\Delta R \\
\Delta R_{j} \\
K \\
\Delta \theta \\
\Delta \theta_{1} \\
\Delta \theta_{2}
\end{array}\right]} \\
+G_{R}\left[\begin{array}{l}
n_{R}(k) \\
n_{b}(k)
\end{array}\right]
\end{gathered}
$$

where $x_{i d}(k), y_{i d}(k)$ are the ideal target positions for the $k$ th measurement. $H_{R}$ is the Jacobian of $f_{\text {Radar }}(\cdot)$ with respect to the vector with bias terms. It is a $6 \times 2$ matrix, whose first two rows are equal, raising a potential observation problem in our bias estimation procedures (our procedures take this fact into account). $G_{R}$ is the Jacobian of Radar(.) with respect to the noise vector. It is a $2 \times 2$ matrix.

There is also a potential time bias, leading to an equivalent position bias aligned with velocity. Then, $(X, Y)$ projected measures will suffer an additional bias of the form

$$
\begin{aligned}
& x_{k, \text { final }}=x_{k}-V_{X} \Delta t, \\
& y_{k, \text { final }}=y_{k}-V_{Y} \Delta t,
\end{aligned}
$$

where $\left(V_{X}, V_{Y}\right)$ are the velocity vectors of the target in this time and $\Delta t$ is the time bias for the radar.

Finally, it should be noted that there are collocated PSRSSR and PSR-mode S radars, for which we will define two virtual sensors, one for PSR and another one for the secondary radar.

\section{2) ADS-B ERROR MODELS}

With precise navigation systems as the ones currently deployed in modern aircraft, ADS-B measures suffer mainly from a time-stamping error that could lead to a time bias, different for each aircraft. Also, a local-to-aircraft position bias is assumed to be potentially present.

The $k$ th position measurement $\left(x_{k}, y_{k}\right)$, obtained using the stereographic projection over latitude, longitude, and height measures, may be modeled as

$$
\begin{aligned}
& x_{k}=x_{i d}(k)-V_{X} \Delta t_{j}+\Delta X_{j}+n_{x}(k), \\
& y_{k}=y_{i d}(k)-V_{Y} \Delta t_{j}+\Delta Y_{j}+n_{y}(k),
\end{aligned}
$$

where $x_{i d}(k), y_{i d}(k)$ are the ideal target positions for the $k$ th measurement, $\left(V_{X}, V_{Y}\right)$ are the velocity vectors of the target in this time, $\left(\Delta X_{j}, \Delta Y_{j}\right)$ are the $X, Y$ bias for the $j$ th aircraft, $\Delta t_{j}$ is the time bias for the $j$ th aircraft, and $n_{x}(k), n_{y}(k)$ are the measurement noise errors.

Finally, we must note the potential observability problem as it is not possible to discriminate position bias along aircraft direction and time bias.
3) WIDE AREA MULTILATERATION ERROR MODELS Wide area multilateration measurement performs time difference of arrival (TDOA) estimation to calculate target position. Irrespective of the method used for position estimation, the basic measures used to obtain aircraft position are the times of arrival of the same signal emitted from this target. The bias of multilateration is a function of several variables, including

- geometry of the receiver(s) and transmitter(s),

- timing accuracy of the receiver system, and

- accuracy of the synchronization of transmitting sites or receiving sites. This can be degraded by unknown propagation effects.

It should be noted that the multilateration system has internal calibration means, because without them no position estimation would be possible. So we are dealing with remaining errors after this calibration. This is a subject still under active research; a description of the main error terms may be found in Leeson [7]. In this system, we assume that the bias changes in space are not too fast, and therefore we perform a discretization of the space in cells. Then, the $k$ th position measurement $\left(x_{k}, y_{k}\right)$, obtained using the stereographic projection over latitude, longitude, and height measures, may be modeled as

$$
\begin{aligned}
& x_{k}=x_{i d}(k)-V_{X} \Delta t+\Delta X(n)+n_{x}(k), \\
& y_{k}=y_{i d}(k)-V_{Y} \Delta t+\Delta Y(n)+n_{y}(k),
\end{aligned}
$$

where $x_{i d}(k), y_{i d}(k)$ are the ideal target positions for the $k$ th measurement, $\left(V_{X}, V_{Y}\right)$ are the velocity vectors of the target in this time, $(\Delta X(n), \Delta Y(n))$ are the $X, Y$ bias for the $n$th cell in the cell list, equal for all aircraft, $\Delta t$ is the time bias for all aircraft and cells, and $\left(n_{x}(k), n_{y}(k)\right)$ are the noise components in the stereographic plane.

\section{4) NOISE ESTIMATION PROCEDURE}

WAM and radar measurement error covariances are calculated by taking sets of three consecutive measurements from a given monosensor track, in not maneuvering segments. With the first and last measure of each set, we perform a linear interpolation for the time of the second measure. Then we perform a difference between this extrapolated measure and the actual measurement taken at that position. This difference error has a covariance related with measurement covariance (assuming it is equal for the three measurements of the same sensor) and with the time differences between the three measures. Performing an adequate transformation over the difference, we obtain a pseudo-measure with the same covariance of the measures. Calculating its covariance, by averaging out results from consecutive differences, we can obtain each sensor covariance. Note that for WAM they must be calculated for each error cell, in the $X-Y$ direction, assuming there is cross covariance, and for ATC radar we can safely assume there is no cross covariance and calculate range and azimuth variances.

\section{5) BiAs ESTIMATION ARCHITECTURE}

From the previous description it is evident that there are three different kinds of bias terms:

- terms dependent on the sensor,

- terms dependent on the sensor-target pair and

- terms dependent only on the target. 
The bias estimation procedure takes this fact into account, and is based on three steps:

- Track bias estimation: From all the available data from a given aircraft, integrated in a multisensor track, an estimation of all the bias terms from all sensors providing measures to this track is obtained. As important as obtaining a vector of bias estimates is obtaining a consistent measurement of its covariance matrix.

- Sensor bias estimation: This method integrates all track bias estimators in an efficient manner, to obtain sensor-related biases. It assumes that the previously described bias vectors are independent measures (as they come from different aircraft measures) of the sensor bias terms.

- Target bias estimation: This is obtained for each aircraft, using the corresponding measurement reports with sensor bias corrected.

\section{C) Fine association}

The first process in fine association is based on merging the short monosensor tracks not associated during gross association with the multisensor tracks. This process also initiates new multisensor tracks with the monosensor tracks not associated to multisensor tracks in previous steps, because they were too short. Those new multisensor tracks will also be used to try and merge other short monosensor tracks not previously associated.

The methods used to perform those mergings are based on position measurement (including barometric and geometric heights) compatibility testing, based on the combination of a linear interpolation in time of measurements from the multisensor and monosensor tracks and a percentage of compatible target reports between the two tracks. To enhance fine association, the method starts with higher quality measures, as are those coming from mode $S, A D S$, or WAM (from mode $S$ signals), and later processes the rest of the sensor types.

Those compatibility tests, if necessary, include height completion procedures to test whether each measure is compatible with the multisensor track under evaluation. The association is a recursive process, as the association of small tracks could lead to multisensor track life extension; therefore all small tracks in the vicinity will have to be re-evaluated after this merging. Internally, after each recursion in the association process, a height completion for the multisensor track is performed, especially important for those data without height (PSR).

Finally, this function also merges pairs of multisensor tracks not associated during gross association, and also those new multisensor tracks created during fine association, using a process analogous to that used for small tracks.

\section{TRAJECTORY SMDOTHING}

In this section, we will describe the new proposed method for trajectory reconstruction. In order to check its performance improvement over other techniques we have taken the performance of two classical approaches as reference.

\section{A) Previous approaches}

Data smoothing has been tackled from different points of view. Splines are a current method for non-parametric data fitting. For instance, one of the reconstruction techniques currently being operated uses beta-splines [8]. However, a theoretically optimal approach may consist in a double tracking loop in the forward and backward directions for smoothing, named as "optimal smoother" $[2,9,10]$, considering detailed models of sensors and aircraft motion.

Two previous reconstruction techniques have been considered. The first one is a suboptimal fixed-interval smoothing algorithm presented by Helmick et al. [g] and the second one is a spline-based reconstruction.

\section{1) FIXED-INTERVAL SMOOTHER}

This is a suboptimal approach based on the use of two Interacting Multiple Mode (IMM) filters. One of the filters propagates in the forward-time direction and the other in the backward-time direction. This algorithm is an approximation that considers only the motion model over two successive sampling periods. The two motion models implemented are constant-velocity models, one with no plant noise and the other with plant noise covariance:

$$
\left[\begin{array}{cccc}
\frac{T^{4}}{4} & \frac{T^{3}}{2} & 0 & 0 \\
\frac{T^{3}}{2} & T^{2} & 0 & 0 \\
0 & 0 & \frac{T^{4}}{4} & \frac{T^{3}}{2} \\
0 & 0 & \frac{T^{3}}{2} & T^{2}
\end{array}\right]
$$

The initial model probabilities in the forward-time filter are $\mu_{\mathrm{oF}}=\left[\begin{array}{ll}0.9 & 0.1\end{array}\right]$ and the model-switching probability matrix is given by

$$
P_{i j}=\left[\begin{array}{ll}
0.95 & 0.05 \\
0.10 & 0.90
\end{array}\right]
$$

The final measurement and its covariance are used to obtain initial position estimates and covariance in the backward-time filter. For this filter, the initial mode probabilities have been chosen to be $\mu_{o B}=\left[\begin{array}{lll}0.9 & 0.1\end{array}\right]$.

\section{2) SPLINES}

The second reconstruction technique is based on a "natural" cubic spline that is directly implemented using a MATLAB function (csaps). This second comparison is provided in order to show the behavior of a non-parametric data fitting with no special tuning.

\section{B) Proposed solution}

Since target behavior may change dramatically (e.g. development of maneuvers inserted between uniform-motion segments), a single kinematic model is insufficient. We have applied an IMM algorithm to model aircraft behavior, a wellreferred algorithm for maneuvering target tracking that dynamically determines the most likely model at each time $[11,12]$. This algorithm has been extensively applied to a type of problem referred to as jump Markov or hybrid-state problem, which involves the joint estimation for both the continuous-valued state vector and a discrete-valued state 
variable, based only in the sequence of available observations. In our case, the state vector contains position and velocity in reconstruction, whereas a discrete variable represents the $\mathrm{MoF}$ at each time, $\{U, T, L\}$, denoting uniform, transversal, longitudinal.

Our approach carries out two stages. The trajectory is segmented in regular motion segments with a first pass to extract the segments. Then, in the second phase, an accurate reconstruction, with less uncertainty, can be performed according to the models identified in the flight and explicitely taking into account the transitions and parameters computed.

The segments are obtained from the IMM mode probabilities computed in a double forward-backward loop. This process provides a first division of sequence of associated measures in MoF segments, but probably with a high rate of over-division produced by noisy transitions. So, a refinement is applied in a second phase. Very short segments are removed, fused with neighbor adjacent segments, and adjacent uniform segments are analyzed to check whether they can be fused. Finally, all MoF segments are validated with a least-squares test. The kinematic parameters corresponding to different types of motion are computed to generate idealized paths, and then the averaged normalized residual is compared with a maximum threshold. If the averaged residual is not acceptable, the segment is labeled as "unknown".

Then, the trajectory interpolation is carried out with an optimal smoothing filter operating forward and backward [2]. It takes into account the advantage of classified MoF segments when available and validated (those accomplishing the least-squares fitting test). The maneuvering parameters describing the "mean" values along the segment are used in the dynamic models, adapted to the specific conditions of the data segment. For instance, in the case of a turn model, a circular prediction model is applied, taking into account the parameters for circle radius and center. Besides, the information about motion intervals is used in the structure. The transition probabilities are modified in the intervals close to edges, and the plant noises are also increased in the presence of close transitions. When no validated segment class is available ("unknown" or "recovered" segment), the prediction filter uses default values and a wider plant noise to avoid degradation. Therefore, all modes exploit information about the parameters used in reconstruction and also whether they are applicable or not.

If available, aircraft derived data (ADD), the velocity data in the target reports from mode $S$ and ADS sensors, are also used in the reconstruction. In this case, the velocity measurements (groundspeed and heading) are projected onto the stereographic plane, together with their error covariance matrix, and integrated in the reconstruction filters through variablesize projection matrices. In order to avoid instabilities due to bad information, a consistency check is performed before integrating this velocity information in the reconstruction. The deviation between estimated and measured velocities is compared with a maximum allowed deviation before acceptance.

\section{RESULTS}

In this section, we present some results to illustrate the performance of the whole trajectory reconstruction system. We decompose the discussion into three sections: bias estimation, association, and reconstruction.

\section{A) Noise and bias estimation}

Next, we describe some noise and bias estimation results from a simulated data example, with error models consistent with the described models. Of course, these results are dependent on the actual position of simulated radars and the relative geometry of targets.

This is a scenario with 61 aircraft with a mixed fleet of SSR and mode $S$ transponders, and mode S, SSR and PSR radars.

In Table 1 we summarize noise and bias estimation results, for selected radars and coordinates. Having the same number and different types means these are different virtual sensors from the same real physical sensor. The advantage of fusing multiple radars and ground sensor data with aircraft-derived data through the mode $S$ link is obtained if the sensor errors are correctly characterized (the biases are correctly estimated and removed and standard deviations are estimated).

Quite often the $95 \%$ band of error in bias estimation is not respected due to

- approximations in the bias processing and

- presence of transponder bias, whose average is mixed with radar bias.

But it is clear from these and other results that the bias estimation is converging to values near the actual ones.

With real data, typical $\mathbf{l} \mathbf{h}$ scenarios tend to have in the order of thousands of aircraft, and typical bias estimation error bands $(95 \%)$ are in the order of several meters.

\section{B) Association}

Here we provide results regarding association for a real scenario, with only $20 \mathrm{~min}$ of data. In this scenario we have mode $S$ and SSR radars, and some of them are collocated. Collocated radars provide the best-quality measure (mode $\mathrm{S}>\mathrm{SSR}>$ PSR) if there are several plots available for an aircraft in the same scan, one of each collocated radar. Therefore misses from a given higher quality mode result in short tracks of lower quality collocated sensors not associated in gross association and recovered quite often in fine association. This can be observed in Table 2 for collocated radars 1,2 , and 3 , where the percentage of correct gross association is much lower for the radar mode (SSR or PSR) with less quality. It should also be noted that the PSR false alarm generation process may lead to higher numbers of non-associated plots.

Due to border effects in a short scenario, these numbers are quite conservative.

Our assessments show three different effects after fine association:

Table 1. Simulated noise and bias estimation results.

\begin{tabular}{lllcll}
\hline $\begin{array}{l}\text { Radar } \\
\text { number } \\
\text { and type }\end{array}$ & $\begin{array}{l}\text { Range } \\
\text { noise } \\
(\mathrm{m})\end{array}$ & $\begin{array}{l}\text { Estimated } \\
\text { range noise } \\
(\mathrm{m})\end{array}$ & $\begin{array}{l}\text { Range } \\
\text { bias }(\mathrm{m})\end{array}$ & $\begin{array}{l}\text { Estimated } \\
\text { range bias } \\
(\mathbf{m})\end{array}$ & $\begin{array}{l}\text { Estimated } \\
\text { range bias } \\
\mathbf{9 5} \% \text { band (m) }\end{array}$ \\
\hline 1-PSR & 110 & 120.40 & 50 & 17.73 & \pm 21.4 \\
2-MS & 74 & 73.79 & 0 & -14.4 & \pm 29 \\
2-SSR & 74 & 78.04 & 0 & -16.71 & \pm 3.4 \\
3-SSR & 74 & 73.60 & 25 & 15.87 & \pm 26.7 \\
4-SSR & 74 & 71.27 & 25 & -16.75 & \pm 26.9 \\
5-MS & 74 & 76.92 & 150 & 163.26 & \pm 27.1 \\
5-SSR & 74 & 72.93 & 150 & 195.01 & \pm 38.1 \\
\hline
\end{tabular}


Table 2. Real data association results.

\begin{tabular}{lcc}
\hline $\begin{array}{l}\text { Radar number } \\
\text { and type }\end{array}$ & $\begin{array}{l}\text { Percentage of } \\
\text { unassociated plots in } \\
\text { gross association (\%) }\end{array}$ & $\begin{array}{l}\text { Percentage of } \\
\text { unassociated plots in } \\
\text { fine association (\%) }\end{array}$ \\
\hline 1-MS & 2.42 & 0.015 \\
1-SSR 1 & 23.97 & 1.37 \\
1-PSR 1 & 88.56 & 59.61 \\
2-MS & 4.9 & 0.029 \\
2-SSR & 27.49 & 0.26 \\
3-SSR & 7.7 & 0.31 \\
3-PSR & 74.3 & 49.29 \\
4-SSR & 14.4 & 1.18 \\
\hline
\end{tabular}

- Most non-associated plots are associated to previously existing tracks, especially at track initiation.

- Short trajectories appear after fine association.

- Bias is corrected, and therefore different sensor measures are better aligned.

\section{C) Smoothing}

In this section, the reconstructions performed on two trajectories using the previously described techniques will be presented. One synthetic trajectory and a real one have been chosen to illustrate the quality of the reconstruction. For the synthetic scenario, we represent the trajectory in Fig. 1 and the absolute value of reconstruction error in Figs 2 and 3 for $X$ and $Y$ coordinates. The synthetic trajectory is used to demonstrate the performance improvement of the proposed method over classical approaches described in Section IV-A. For the real scenarios, as there is no ground truth the measurements and the output of the model-based reconstruction are shown. In order to analyze more in depth, several maneuvers have been zoomed in, which allows seeing the quality of the reconstruction (Figs 4-6).

The synthetic scenario consists of an aircraft that performs a left turn after a straight segment which is continued by another straight segment. It goes from the west to the east at a constant velocity of $300 \mathrm{~m} / \mathrm{s}$.

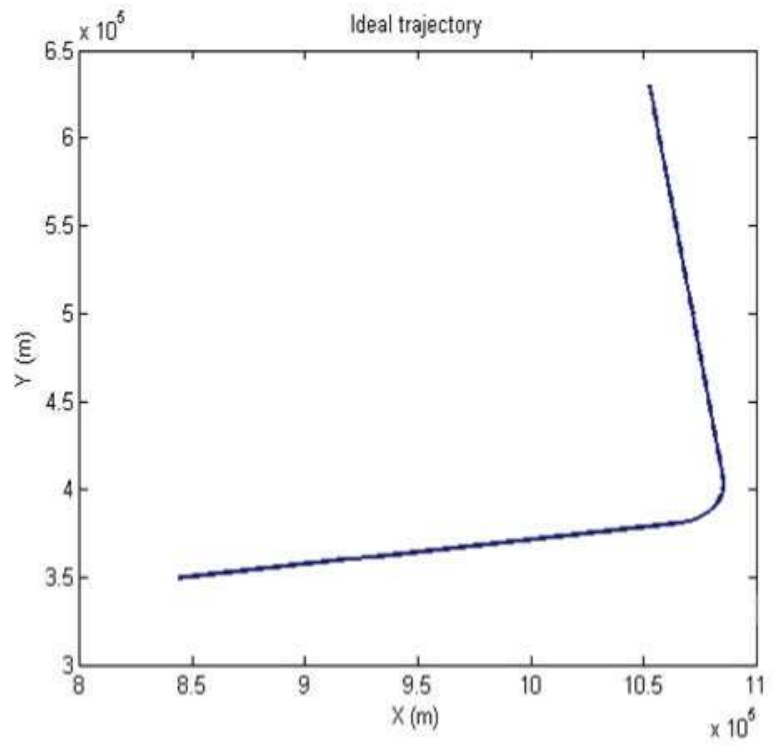

Fig. 1. Ideal trajectory.

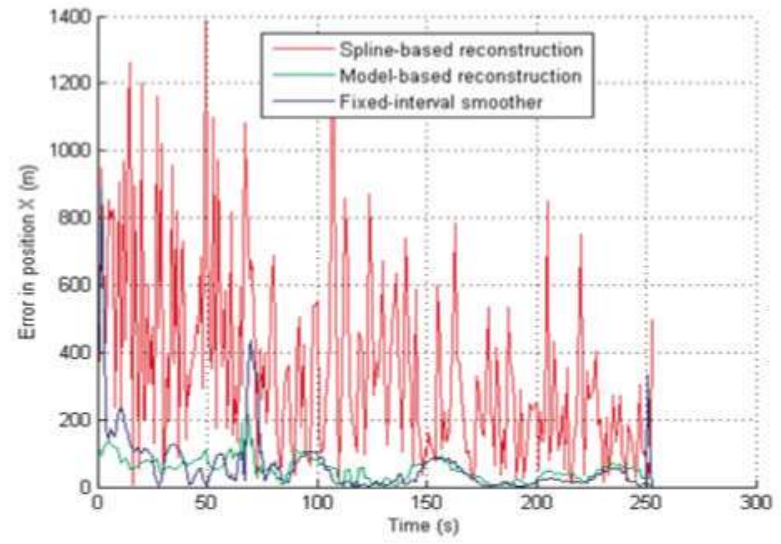

Fig. 2. Error in position, $\mathrm{X}$ coordinate.

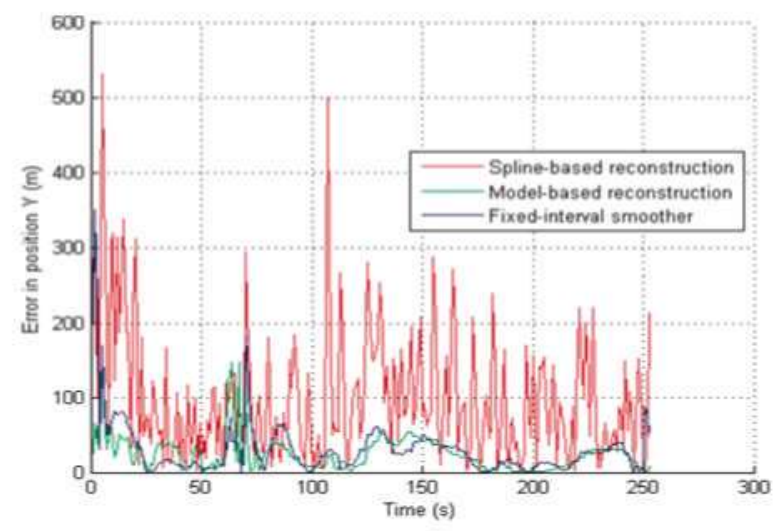

Fig. 3. Error in position, $\mathrm{Y}$ coordinate.

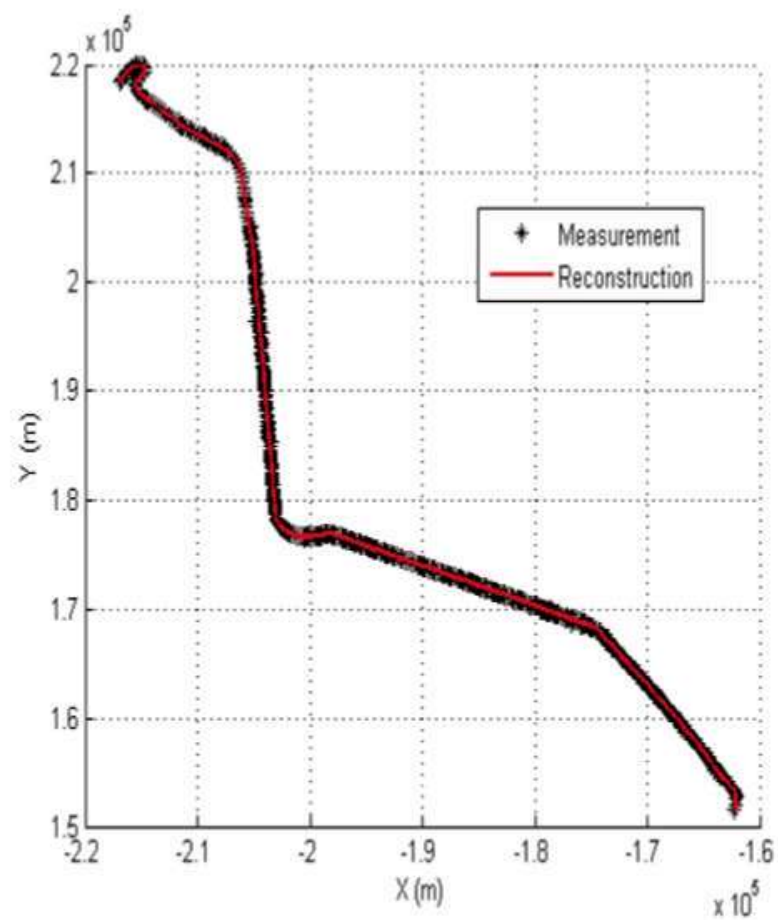

Fig. 4. Measurements and reconstructed real trajectory. 


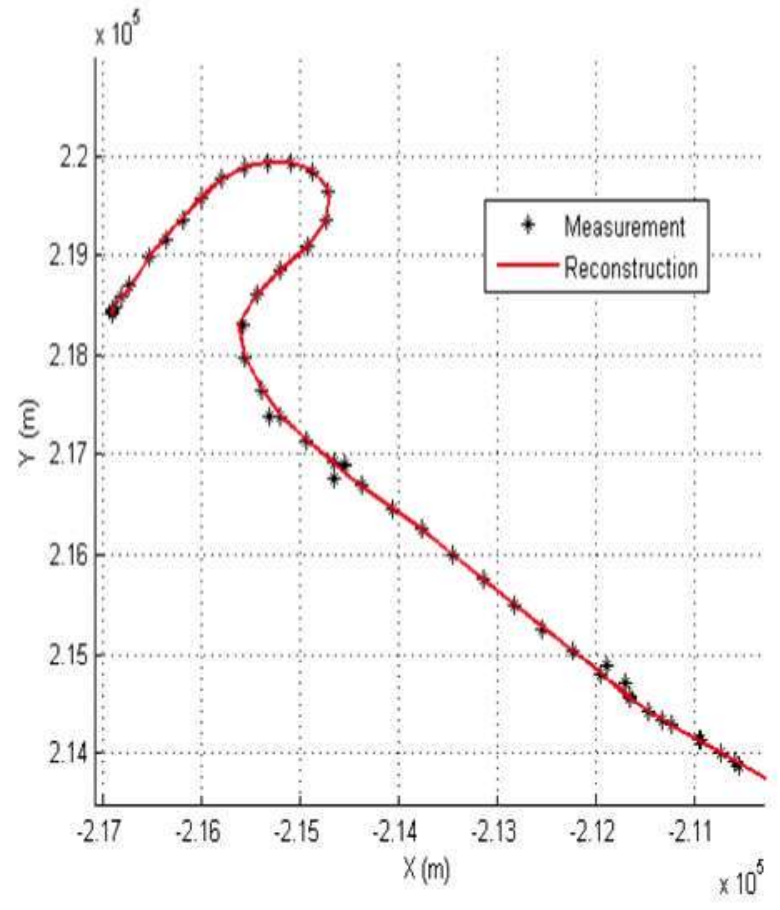

Fig. 5. First detail of reconstructed real trajectory.

The absolute position error in the reconstruction is shown in Figs 2, 3 for three methods: spline reconstruction, fixed-interval smoother, and our model-based reconstruction. As can be seen, the output of the parametric approaches is better than the one provided by the spline one. It can be seen that the output of the model-based approach has less error peak than the fixed-interval smoother.

For the real scenario, as can be seen in Figs 4-6, the quality of the reconstruction is more than reasonable, being robust enough to cope with outliers as can be observed in Fig. 5 . However, the reconstruction can be improved by parameter tuning, which implies a trade-off between noise reduction and maneuver response.

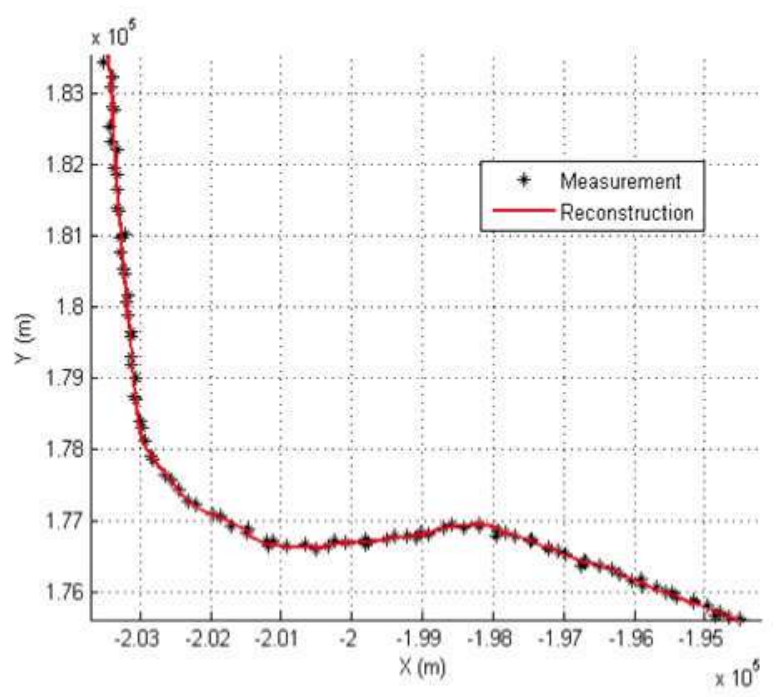

Fig. 6. Second detail of reconstructed real trajectory.

\section{CONCLUSIONS}

A new processor for ATC offline multisensory data processing has been designed. Its architecture, although similar to ATC real-time processes, takes advantage of the lack of real-time constraints and performs a smoothing process instead of a filtering one. Extensive real and simulated data results show the capability of this system to generate high-accuracy smoothed trajectories. These will serve as reference trajectories for the evaluation of operative ATC data processors (comparing output tracks of data processors with the reference trajectory).

The proposed system is capable of processing data for the type of sensors used for ATC now and in the near future (mode S radar, enhanced surveillance, SSR, PSR, WAM, and ADS-B). For this purpose, the system incorporates new methods to correct sensor bias for all kinds of sensors, to perform a high-quality association, and, finally, for reconstruction of high-accuracy trajectories using the measurements of many dissimilar sensors.

\section{ACKNOWLEDGMENTS}

This work was funded by contract EUROCONTROL's TRES, CICYT TSI2005-07344, CICYT TEC2005-07186, CAM MADRINET S-0505/TIC/0255.

\section{REFERENCES}

[1] Besada, J.; Soto, A.; de Miguel, G.; Garcia, J; Alcazar, R.; Voet, E.: TRES: multiradar-multisensor data processing assessment using opportunity, in 2008 IEEE Radar Conf., 2008.

[2] Gelb, A.: Applied Optimal Estimation, The M.I.T. Press, Cambridge, 1982.

[3] Rafati, A.; Moshiri, B.; Salahshoor, K.; Tabatabaei, M.: Asynchronous Sensor Bias Estimation in Multisensor-Multitarget Systems. 2006 IEEE International Conference on Multisensor Fusion and Integration for Intelligent Systems., 2006.

[4] Lin, X.; Bar-Shalom, Y.; Kirubarajan, T.: Multisensor multitarget bias estimation for general asynchronous sensors. IEEE Trans. Aerosp. Electron. Syst., 41 (2005), 899-921.

[5] Pyung, S.K.: Separate-bias estimation scheme with diversely behaved biases. IEEE Trans. Aerosp. Electron. Syst., 38 (2002), 333-339.

[6] Paradowski, L; Kowalski, Z: An effective coordinates conversion algorithm for radar-controlled anti-aircraft systems, in 12th Int. Conf. Microwaves and Radar, MIKON '98, 1998.

[7] Leeson, M.J.: Error analysis for a wide area multilateration system QinetiQ/ C\&IS/ ADC/ 520896/7/19.

[8] Renes, J.J.; Best, M.R.: MURATREC Multi-Radar Trajectory Reconstruction Facility, Theoretical Background Technical Report NRL CR 89196 L, National Luchten Ruimtevaartlaboratorium, 1985.

[9] Helmick, R.E.; Blair, W.D.; Hoffman, S.A.: Fixed-interval smoothing for Markovian switching systems. IEEE Trans. Inf. Theory, 41 (1995), 1845-1855.

[10] García, J.; Molina, J.M.; Besada, J.; de Miguel, G.: Model-based trajectory reconstruction using IMM smoothing and motion pattern identification, in Proc. 1oth Int. Conf. Information Fusion, 2007.

[11] Bar-Shalom, Y.: Multitarget-Multisensor Tracking: Advanced Applications, Artech House, Norwood, MA, 1990.

[12] Rong Li, X.; Bar-Shalom, Y.: Design of an interacting multiple model algorithm for air traffic control tracking. IEEE Trans. Control Syst. Technol., 1 (1993), 186-194. 


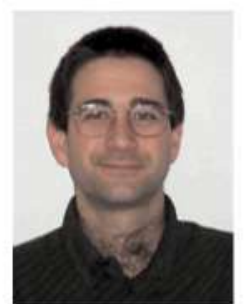

Jesus Garcia is currently an Associate Professor at the Universidad Carlos III de Madrid and has been a member of the Computer Science Department since 2000. He received his BS in Telecommunications Engineering from Universidad Politécnica de Madrid in 1996, and his Ph.D. degree from the same university in 2001 . He is currently working in the Applied Artificial Intelligence Research Group. Before, he was a member of the Universidad Politécnica de Madrid's Data Processing and Simulation Group. He has participated in several national and European projects related to Air Traffic Management. His main interests are artificial intelligence applied to engineering in the context of radar and image data processing, machine vision, and air traffic management.

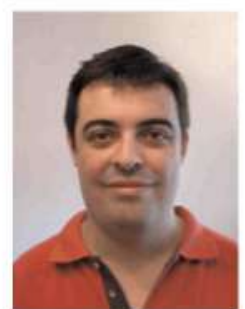

Juan A. Besada received a degree in Telecommunication Engineering from the Universidad Politecnica de Madrid in 1996 and a Ph.D. from the same university in 2001. He has worked in the Signal Processing and Simulation Group of the same university since 1995 , participating in several national and European projects related to Air Traffic Control. He is currently Associate Professor at Universidad Politecnica de Madrid. His main interests are air traffic control, navigation, and data fusion.

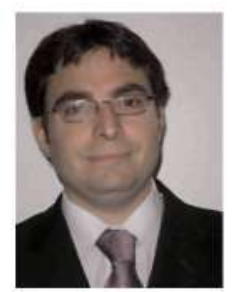

Andres Soto received a degree in Telecommunication Engineering from the Universidad Politecnica de Madrid (UPM) in 2002 and is now pursuing a Ph.D. at the same university. He is currently working at INDRA SISTEMAS developing the next generation of Flight Data Processing systems in what is known as the iTEC project. Previously, he worked for the Signal Processing and Simulation Group of the UPM for 5 years. His main interests are radar data processing, air traffic control, and data fusion.

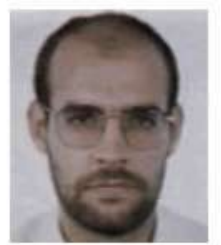

Gonzalo de Miguel received his Telecommunication Engineering degree in 1989 and his Ph.D. in 1994 from the Universidad Politécnica de Madrid. He is currently a Professor at the Department of Signals, Systems and Radiocommunications of this university and is a member of the Data Processing and Simulation Research Group at the Telecommunication School. His fields of interest and activity are radar signal processing and data processing for air traffic control applications. 